\title{
DETERMINATION OF THE TOTAL PHENOLIC AND TOTAL FLAVONOID CONTENTS IN CYNARA SCOLYMUS L. EXTRACTS
}

\section{(C) Ciobanu Cristina}

State Medical and Pharmaceutical University "Nicolae Testemițanu", Faculty of Pharmacy, Chisinau, Republic of Moldova

Artichoke - Cynara scolymus L. is a plant widely cultivated in Europe and the United States effective on the treatments of hepatobiliary diseases, hyperlipidaemia and cholesterol metabolism (1). The purpose of the study was to determine the main biological active compounds in leaf and flower heads extracts obtained from artichokes grown in Centre for the Cultivation of Medicinal plants of The State Medical and Pharmaceutical University "Nicolae Testemiţanu". Determination of total phenol and total flavonoid contents in C. solymus $\mathrm{L}$. leaves and flower heads extracts in different concentrations of the ethanol solvent used was carried out using spectrophotometric methods. The total phenolic content was determinated by using cynarin (1,3-dicaffeoylquinic acid) as reference material, in the $360 \mathrm{~nm}$ wavelength, calibration curve for the analyte was linear with the correlation coefficients $(y=51,572 x, R 2=0.9973)$. Total flavonoid determination was performed by using rutin as a standard, in the $430 \mathrm{~nm}$ wavelength, the concentrations of flavonoid in the test samples were calculated from the calibration plot of rutin $(y=36.985 x, R 2=0.9994)$. The results showed that the contents of total polyphenols and total flavonoids were highest $70 \%$ ethanol solvent for both leaf and flower heads extracts. Results of the analysis revealed that artichoke leaf extract contains $0.72 \%$ flavonoids, $1.27 \%$ polyphenols; flower heads extract contains $0.50 \%$ flavonoids and $1.04 \%$ polyphenols, respectively. The value of total phenolic and total flavonoid content of artichoke leaf and flower heads extracts demonstrated that flower heads of $C$. solymus L. may be used as alternative raw materials in obtaining artichoke plant extracts.

Reference: (1) Löhr G, Deters A, Hensel A. In vitro investigations of Cynara scolymus $L$. extract on cell physiology of HepG2 liver cells. Braz J Pharm Sci 2009; 45: 201-208.

\section{BIOLOGICAL ASPECTS OF CYNARA SCOLYMUS L. CULTIVATED IN THE REPUBLIC OF MOLDOVA}

\section{() Ciobanu Cristina}

State Medical and Pharmaceutical University "Nicolae Testemițanu", Faculty of Pharmacy, Chisinau, Republic of Moldova

Artichoke $C$. scolymus $L$. is a medicinal plant frequently used in folk and scientific medicine due to its therapeutic properties, including antioxidant effects, hepatoprotective ability and cholesterol-lowering effects. The aim of the work was to evaluate the morphoanatomical adaptations of the artichoke to the environmental conditions of the Republic of Moldova that differ from countries of plant origin. The morpho-anatomical investigations were effectuated on the artichokes grown in the Collection of Medicinal Plants of the Centre for the Cultivation of Medicinal plants of our University. Were carried out morpho-biometrical evaluation of the artichoke plants and anatomical study of their leaves. The morpho-biometrical evaluation of the artichoke was effectuated on more that 10 parameters: plant height, steam diameter, number of branches, foliar levels, num- ber of leaves on a plant, leaf length, leaf width, number of leaf lobes, flower heads on a plant, flower heads on a branch, flower heads diameter, also leaf area was estimated. The statistic evaluation shown that the principle biometrical parameters are average plant height $85,5 \pm 5,2 \mathrm{~cm}$ foliar levels $-12,0 \pm 0,8$ number of leaves on a plant $-20,0 \pm 1,1$ average leaf area $-146,7 \mathrm{~cm}$. Anatomical study of artichoke leaf elucidated specific anatomical structures: glandular and 2 types of covering trichomes on both epiderma, dorsoventral structure of mesophyll, anomocytic stomata, collateral vascular bundles and angular collenchyma. In conclusion the morpho-biometric results in correlation with anatomical data demonstrate that C.scolymus L. grow and develop well in the climatic conditions of Republic of Moldova and can be targeted for further phytochemical analysis. 